\title{
Association of peripheral hyperopia with axial elongation in high myopia
}

\author{
Zhen-Yong Zhang $\cdot$ Xing-Ru Zhang $\cdot$ Zhi-Qiang Yu
}

Received: 9 January 2013 / Accepted: 23 January 2013 / Published online: 12 February 2013

(C) Springer-Verlag Berlin Heidelberg 2013

\section{Dear Editor:}

Saka and associates, in a prospective longitudinal study, have investigated the changes in axial length in adults with high myopia and observed that the myopic eyes continue to elongate during a 2-year period of follow-up [1]. Despite the universal acceptance that axial length of the eye reaches adult level and keeps relatively stable during the 2 nd decade of life, this may not be the case in eyes with myopia, as once myopia is developed, progression can continue throughout childhood and adulthood, particularly in high myopia as in this study.

To date, the mechanism leading to axial elongation and myopia progression remains inconclusive with hyperopic defocus that presumably results from intensive near work and/or accommodation lag, which is considered to be one of the important risk factors. This hyperopic defocus, which is often referred to as the on-axis defocus, does not appear to be responsible for the axial elongation in this study, because no data is available to support its existence in the subjects. Given that it is more likely for adults with high myopia, who constitute the sampled population, to wear spectacle lenses other than other corrections, peripheral hyperopia may exist in these subjects, as previous studies [2-4] have demonstrated that correcting the on-axis refractive error in moderate to high myopia with conventional spectacle lenses results in hyperopic defocus in the peripheral retina, for which a greater magnitude may appear when considering the fact that the myopic eye per se has already shown a relative hyperopic shift in the periphery [5]. On-axis refraction is of great importance

\section{Z.-Y. Zhang $\cdot$ X.-R. Zhang}

Department of Ophthalmology, Putuo Hospital, Shanghai

University of Chinese Traditional Medicine, Shanghai, China

\section{Z.-Q. Yu $(\bowtie)$}

Department of Ophthalmology, Eye \& ENT Hospital, Fudan

University, No. 19, Baoqing Road,

Shanghai 200031, China

e-mail: yu_zhiqiang0813@163.com in the development or progression of myopia; however, with the understanding that the peripheral retina controls the eye growth [6], off-axis hyperopia is currently believed to exert quite an effect on the development of myopia [7]. Accordingly, the peripheral hyperopia resulting from spectacle lenses for high myopia correction may be a possible explanation for the observations of axial elongation in this study.

The comments above are based on the validity of this study. However, the shortened axial length in 27 eyes at endpoint, which is simply attributed to technical measurement error in this article, would in turn decrease the reliability of this study. More should be done to make a stronger conclusion.

Competing interests No authors have any conflict of interest.

\section{References}

1. Saka N, Moriyama M, Shimada N, Nagaoka N, Fukuda K, Hayashi K, Yoshida T, Tokoro T, Ohno-Matsui K (2012) Changes of axial length measured by IOL master during 2 years in eyes of adults with pathologic myopia. Graefes Arch Clin Exp Ophthalmol Jun 8 [Epub ahead of print]. doi:10.1007/s00417-012-2066-9

2. Charman WN, Radhakrishnan H (2010) Peripheral refraction and the development of refractive error: a review. Ophthalmic Physiol Opt 30:321-338

3. Backhouse S, Fox S, Ibrahim B, Phillips JR (2012) Peripheral refraction in myopia corrected with spectacles versus contact lenses. Ophthalmic Physiol Opt 32:294-303

4. Chen X, Sankaridurg P, Donovan L, Lin Z, Li L, Martinez A, Holden B, Ge J (2010) Characteristics of peripheral refractive errors of myopic and non-myopic Chinese eyes. Vis Res 50:31-35

5. Ehsaei A, Mallen EA, Chisholm CM, Pacey IE (2011) Crosssectional sample of peripheral refraction in four meridians in myopes and emmetropes. Invest Ophthalmol Vis Sci 52:7574-7585

6. Morgan IG, Ohno-Matsui K, Saw SM (2012) Myopia. Lancet 379:1739-1748

7. Smith EL 3rd (2011) Prentice Award Lecture 2010: a case for peripheral optical treatment strategies for myopia. Optom Vis Sci 88:1029-1044 